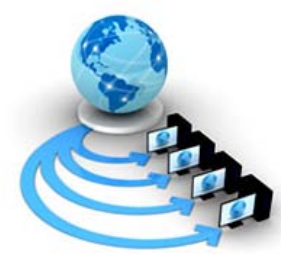

International Journal of Advanced Research in Computer Science

RESEARCH PAPER

\author{
Available Online at www.ijarcs.info
}

\title{
A MULTI-LEVEL VISUAL TRACKING ALGORITHM FOR AUTONOMOUS VEHICLES
}

\author{
K. Narsimlu \\ Ph.D. Research Scholar \\ Dept. of CSE \\ JNT University \\ Hyderabad, India \\ Devendra Rao Guntupalli \\ Senior Vice President \\ Information Systems \\ Cyient Ltd \\ Hyderabad, India
}

\author{
Dr. T. V. Rajini Kanth \\ Professor \\ Dept. of CSE \\ SNIST \\ Hyderabad, India
}

\author{
Anil Kuvvarapu \\ M.S. Student \\ Dept. of CS \\ University of Michigan \\ Michigan, USA
}

\begin{abstract}
A multi-level visual tracking algorithm is proposed for autonomous vehicles based on mean-shift algorithm, cam-shift algorithm and extended kalman filter estimator. The proposed multi-level visual tracking algorithm is implemented and included in simulation to check its performance. Simulation results are captured after applying existing algorithms, proposed multi-level visual tracking algorithm and observed their performance. The simulated results show that the proposed multi-level visual tracking algorithm identifies and tracks the ground moving target efficiently.
\end{abstract}

Keywords: Autonomous Vehicles; Cam-Shift; Extended Kalman Filter; Mean-Shift; Multi-level Visual Tracking Algorithm; Probability Density Function; Retinex Algorithm.

\section{INTRODUCTION}

The autonomous vehicles are commonly known as Unmanned Aerial Vehicles (UAVs), Unmanned Ground Vehicles (UGVs), and Autonomous Underwater Vehicles (AUVs).

The UAVs are commonly used in surveillance and situational awareness applications. The UAVs are very useful in environment monitor where humans are not accessible. These UAVs are also useful for military applications [1] and civil applications [2].

The autonomous visual tracking algorithm is an estimation of a moving target path in an image plane [3]. These visual tracking algorithms can be categorized based on the representation of target shape and selection of target feature.

The representation of target shape uses the target points, target shapes, target silhouette, target contour and target skeletal. The selection of target feature uses the target color, target edge, target texture.

The detection of target uses target background subtraction, target optical flow and target segmentation. The tracking of target uses kernel tracking, point tracking, and silhouette tracking.

The main challenges of target tracking [4], [5], [6], [7] are as follows: 1) target lost, 2) background objects are moving along with the target, 3) light brightness changes on the target, 4) noise in the image.

An efficient multi-level visual tracking algorithm is proposed for target tracking to overcome the target tracking challenges. The process of proposed multi-level visual tracking algorithm is as follows: 1) acquisition, 2) preprocessing, 3) execute the multi-level visual tracking algorithm, 4) post-processing.

The template matching of image processing algorithm is used to detect the Ground Stationary Target (GST) or Ground Moving Target (GMT).

The main motivation is to design and develop an efficient multi-level visual tracking algorithm for UAVs. This algorithm identifies the GST or GMT and tracks the GMT autonomously. The On-board Autonomous Visual Tracking System (AVTS) contains the proposed multi-level visual tracking algorithm.

The main goal of autonomous visual tracking of UAV is to monitor the environment where humans are not accessible [8], [9], [10].

The main purpose of on-board multi-level visual tracking algorithm is to develop and demonstrate the ability to autonomously tracking of GMT from UAV without Ground Control System (GCS) support [11], [12], [13], [14], [15], [16], [17], [18].

The steps of proposed multi-level visual tracking algorithm: 1) GMT selection, 2) GMT detection, 3) GMT tracking, are as shown in Fig. 1.

In this paper, sections are: Section 2 Existing Tracking Algorithms, Section 3 Proposed Multi-level Visual Tracking Algorithm, Section 4 On-board AVTS, Section 5 Simulation Results and Analysis, Section 6 Conclusions. 


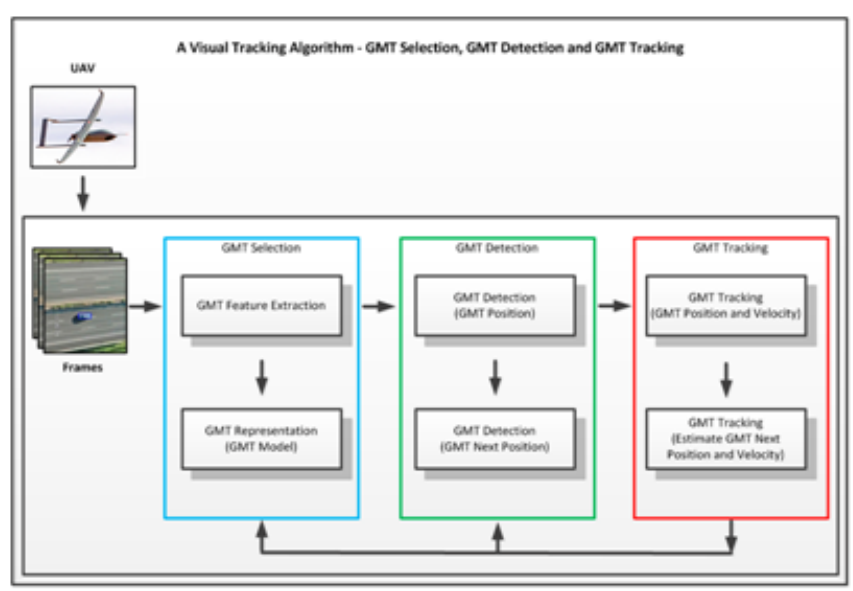

Fig. 1. Proposed Multi-level Visual Tracking Algorithm Steps: GMT Selection, GMT Detection and GMT Tracking.

\section{EXISTING TRACKING ALGORITHMS}

This section explains the existing tracking algorithms [19], [20], [21], [22], [23] in detail.

The various visual tracking algorithms are Mean-Shift (MS) algorithm, Cam-Shift (CS) algorithm, Extended Kalman Filter (EKF) estimator.

A. MS Algorithm

This MS algorithm identifies and tracks the GMT based on Red-Green-Blue (RGB) color histogram Probability Density Function (PDF) of frame [24], [25], [26], [27], [28], [29], [30].

B. CS Algorithm

This CS algorithm identifies and tracks the GMT based on Hue-Saturation-Value (HSV) color histogram new PDF of each frame [31], [32], [33], [34], [35], [36].

C. EKF Estimator

This EKF estimator predicts the GMT position based on GMT previous position [37], [38], [39], [40], [41].

The main problems of the existing GMT tracking algorithms [42], [43], [44], [45], [46], [47], [48], [49], [50] are as follows: 1) GMT lost 2) background GMTs are moving along with the GMT 3) light brightness changes on the GMT 4) noise in the image.

An efficient multi-level visual tracking algorithm is proposed for GMT tracking to overcome the existing GMT tracking problems.

\section{PROPOSED MULTI-LEVEL VISUAL TRACKING ALGORITHM}

This section explains the proposed multi-level visual tracking algorithm in detail.

The process of the proposed multi-level visual tracking algorithm is as follows:

- Acquisition: acquire image frame-by-frame.

- Pre-processing: image enhancement using Retinex algorithm.

- Execute proposed algorithm: execute proposed multilevel visual tracking algorithm.

- Post-processing: export image frame-by-frame to graphs and reports.
The proposed multi-level visual tracking algorithm steps are:

Algorithm 1: Multi-level Visual Tracking Algorithm

Step 1: Source frame (s: 0 to 11 frames) = $f_{s}\left(x_{i}, y_{j}\right)$.

Step 2: Reference frame (s: 0 frame) $=f_{0}\left(x_{i}, y_{j}\right)$

Last Frame (s: 11 frame) $=f_{11}\left(x_{i}, y_{j}\right)$.

Step 3: Select frame $f_{0}\left(x_{i}, y_{j}\right)$.

Step 4: Enhance frame $f_{0}\left(x_{i}, y_{j}\right)$ using Retinex algorithm.

Step 5: Select object frame $f_{o b j}\left(X_{i}, y_{j}\right)$ of the GMT from $f_{0}\left(x_{i}, y_{j}\right)$ frame.

Step 6: Compute $f_{\text {obj }}\left(x_{i}, y_{j}\right)$ object frame PDF.

Step 7: Calculate object frame centre using MS algorithm:

$$
\left.\begin{array}{c}
f_{o b j-c}\left(x_{o b j-c}, y_{o b j-c}\right)= \\
\sum_{i} \sum_{j} x_{i}^{*} f\left(x_{i}, y_{j}\right) \\
\sum_{i} \sum_{j} f\left(x_{i}, y_{j}\right)
\end{array} \frac{\sum_{i} \sum_{j} y_{j}^{*} f\left(x_{i}, y_{j}\right)}{\sum_{i} \sum_{j} f\left(x_{i}, y_{j}\right)}\right)
$$

Step 8: Compute $f_{t}\left(X_{i}, y_{j}\right)$ GMT frame PDF.

Step 9: Calculate target centre using MS algorithm:

$$
\left.\begin{array}{c}
f_{t-c}\left(x_{t-c}, y_{t-c}\right)= \\
\sum_{i} \sum_{j} x_{i}^{*} f\left(x_{i}, y_{j}\right) \\
\sum_{i} \sum_{j} f\left(x_{i}, y_{j}\right)
\end{array} \frac{\sum_{i} \sum_{j} y_{j}^{*} f\left(x_{i}, y_{j}\right)}{\sum_{i} \sum_{j} f\left(x_{i}, y_{j}\right)}\right)
$$

Step 10: IF $\left(\left(f_{t-c}\left(X_{t-c}, y_{t-c}\right)<10\right)\right.$ OR (PDF of $\left.\left.f_{t}\left(x_{i}, y_{j}\right)<0.16\right)\right)$ THEN

$$
f_{o b j-c}\left(x_{o b j-c}, y_{o b j-c}\right)=f_{t-c}\left(x_{t-c}, y_{t-c}\right)
$$

GO TO Step 8

ELSE

Compute GMT frame centre using EKF estimator:

$$
f_{t-c}\left(X_{t-c}, y_{t-c}\right)=\mathrm{A}^{*} f_{o b j-c}\left(X_{o b j-c}, y_{o b j-c}\right)
$$

ENDIF

Step 11: Move the object frame using CS algorithm:

$$
f_{o b j-c}\left(x_{o b j-c}, y_{o b j-c}\right)=f_{t-c}\left(x_{t-c}, y_{t-c}\right)
$$


Step 12: IF (target frame $f_{t}\left(X_{i}, y_{j}\right)==$ last frame

$$
\begin{aligned}
& \left.f_{11}\left(x_{i}, y_{j}\right)\right) \text { THEN } \\
& \text { STOP } \\
& \text { ELSE } \\
& \text { GO TO Step } 8 \\
& \text { ENDIF }
\end{aligned}
$$

The MS and CS algorithms are computationally efficient. But, it is difficult to track GMT when the GMT moves out of frame. Hence, MS and CS with EKF based multi-level visual tracking algorithm, is proposed for GMT tracking.

Whenever the GMT moves out of frame or PDF value more than 0.16 (GMT lost by MS algorithm), then CS algorithm takes the current state of GMT and tracks the GMT continuously. Whenever the GMT moves out of frame or new PDF value more than 0.16 (GMT lost by CS algorithm), then EKF estimates the GMT position and tracks the GMT continuously.

The proposed multi-level visual tracking algorithm data flow diagram is shown in Fig. 2.

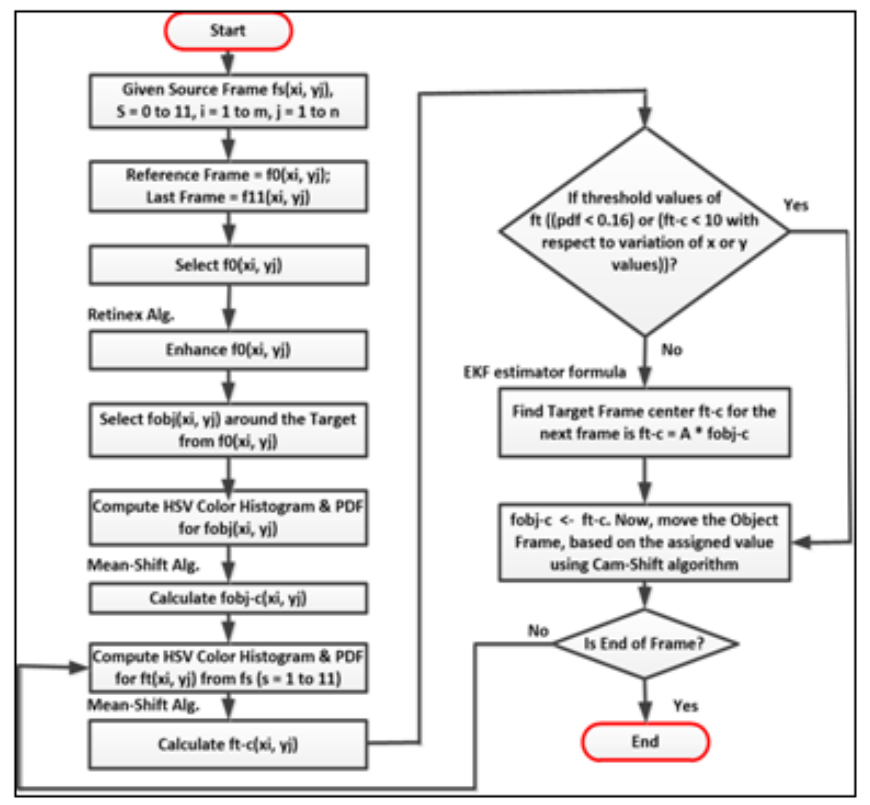

Fig. 2. Proposed Multi-level Visual Tracking Algorithm Data Flow Diagram.

\section{ON-BOARD AVTS}

The On-board AVTS contains gimbaled camera along with proposed multi-level visual tracking algorithm, INS/GPS, UAV guidance [51], [52], [53], camera control [54], [55], [56] and autopilot [57], [58], [59].

\section{SIMULATION RESULTS AND ANALYSIS}

A MATLAB based simulation is developed for determining the proposed multi-level visual tracking algorithm performance. A Simulation of the On-board AVTS is shown in Fig. 3.

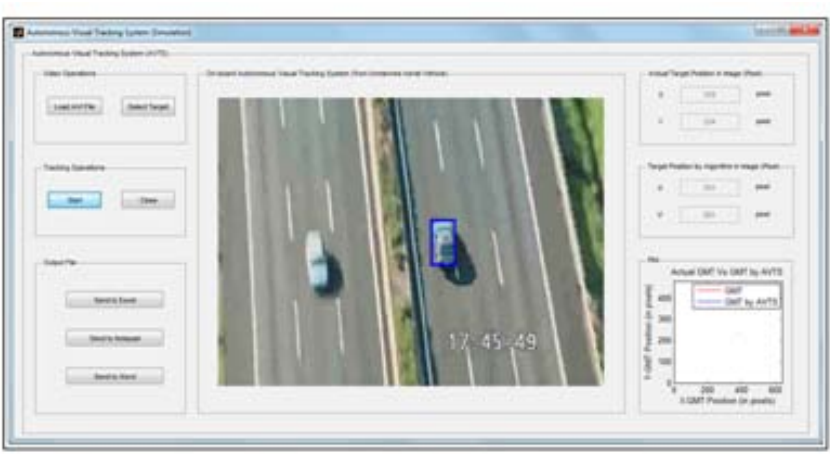

Fig. 3. On-board AVTS Simulation.

This On-board AVTS simulation acquires the image, enhances the image using a Retinex algorithm, executes the proposed multi-level visual tracking algorithm and exports the data to the graphs and reports.

We have provided the aerial input video [60] as an image frames to an On-board AVTS for GMT tracking realtime simulation, are as shown in Fig. 4.

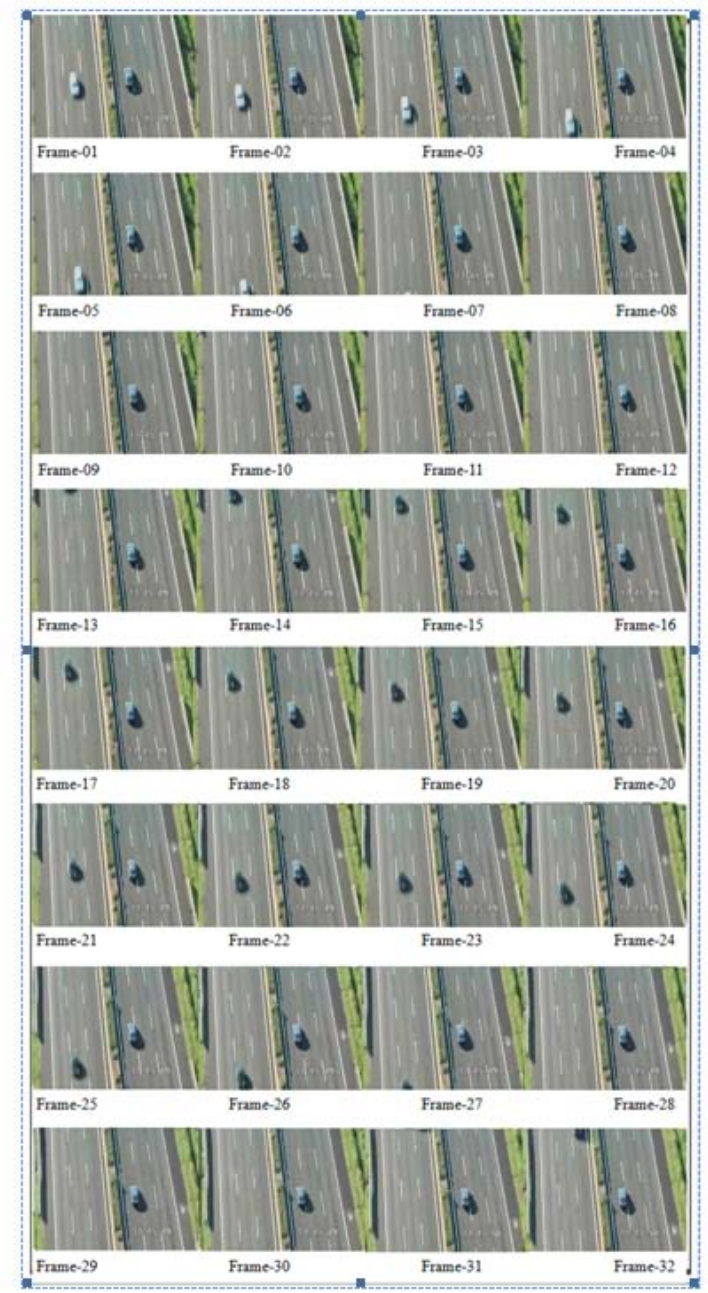

Fig. 4. Input Image Frames (Frame-by-Frame).

The pre-processed image frames using Retinex algorithm are shown in Fig. 5. 


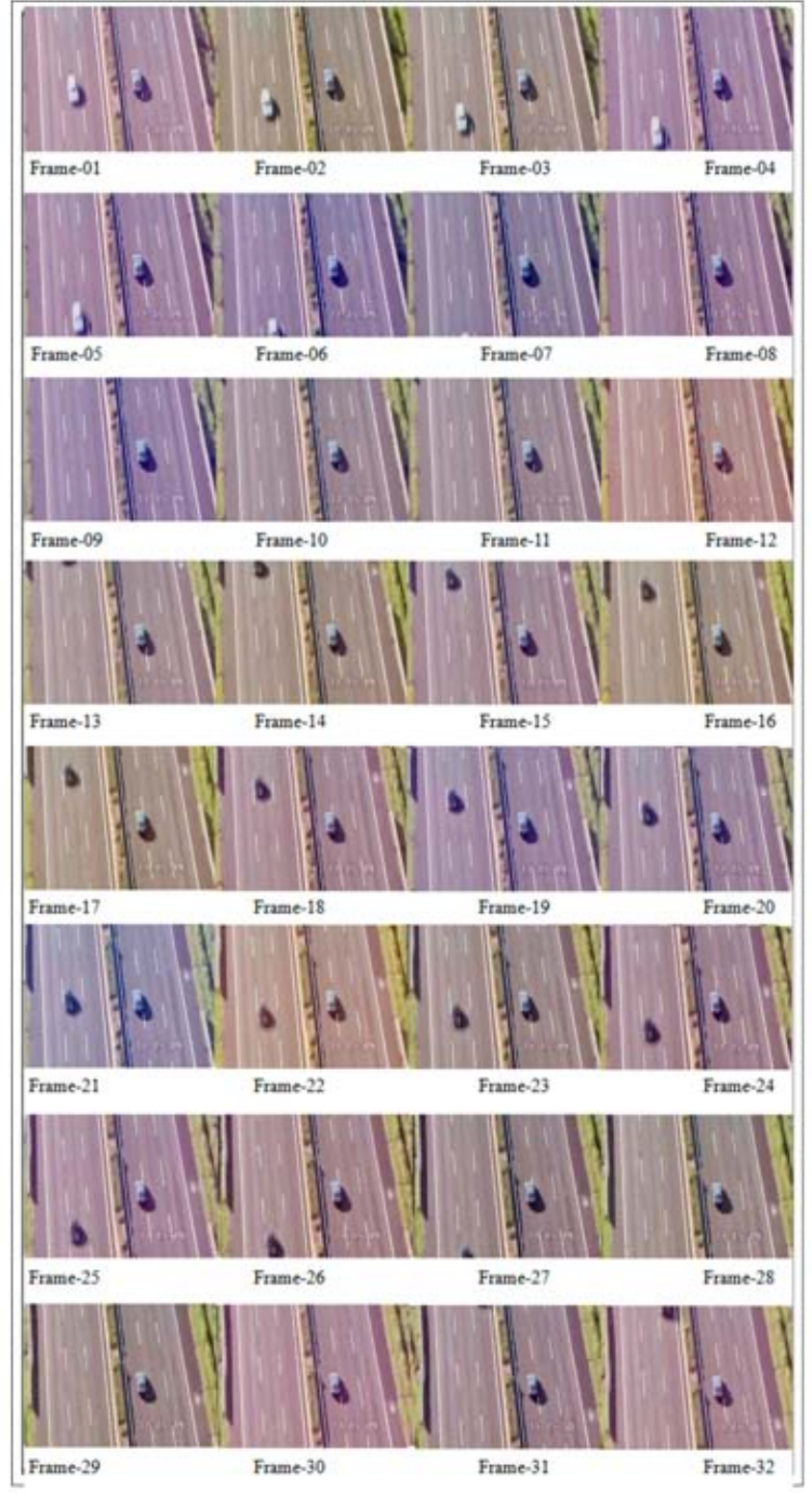

Fig. 5. Pre-processed Image Frames (Frame-by-Frame) using Retinex Algorithm.

The GMT tracking using proposed multi-level visual tracking algorithm is shown in Fig. 6.

We have considered the thirty-two input image frames (resolution is 640x480 pixels) with an On-board AVTS for experimental analysis.

The computed error between the MS algorithm, CS algorithm, EKF estimator and proposed multi-level visual tracking algorithm, are as shown in a Table I.

The computed GMT pixel position by MS algorithm, CS algorithm, EKF estimator and proposed multi-level visual tracking algorithm, are exported for off-line analysis.

The exported results are as shown in Fig. 7.

The execution times between the MS algorithm, CS algorithm, EKF estimator and proposed multi-level visual tracking algorithm are shown in a Table II.

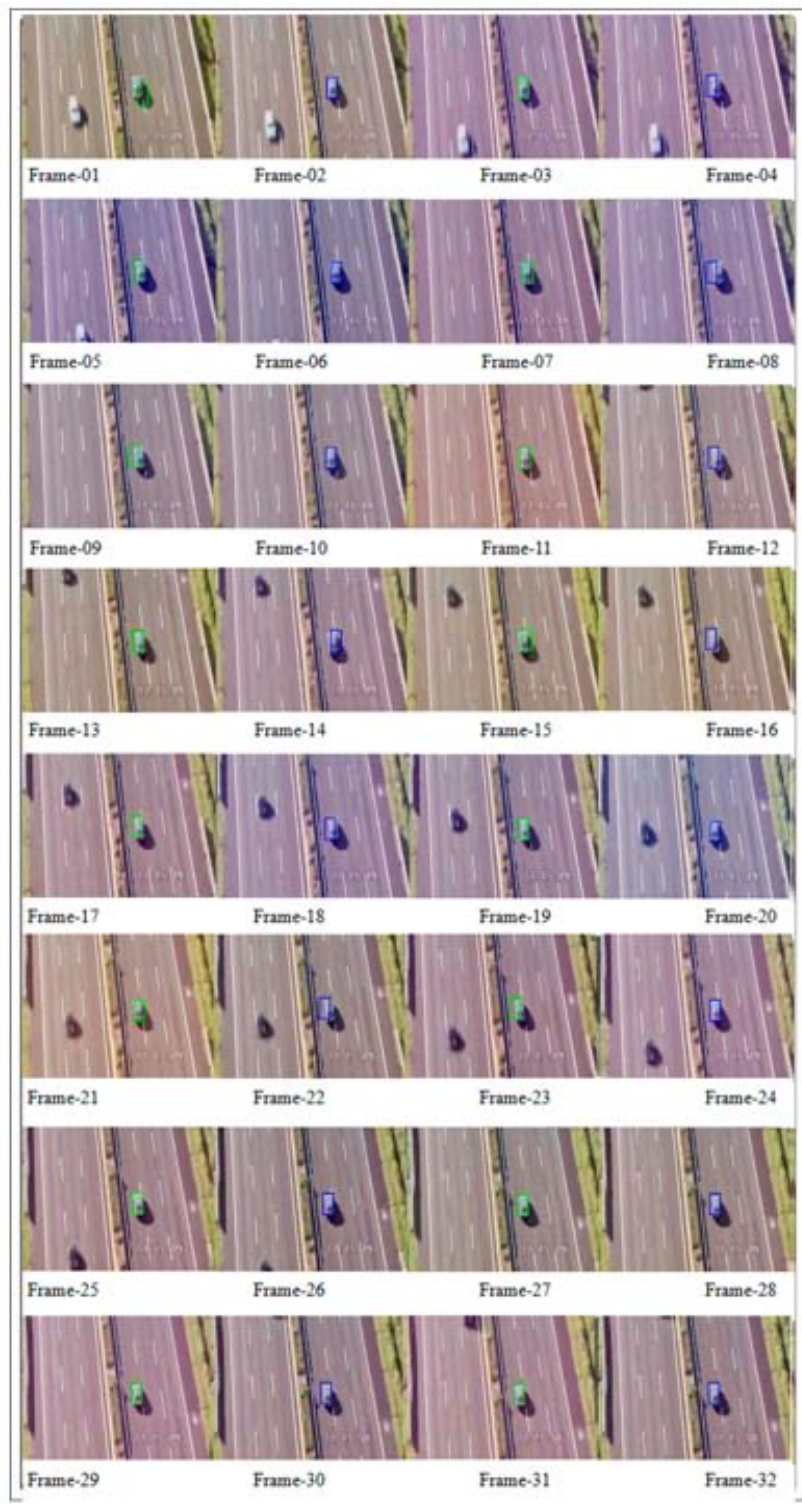

Fig. 6. GMT Tracking (Frame-by-Frame) using Proposed Multi-level Visual Tracking Algorithm.

The proposed multi-level visual tracking algorithm tracks the GMT more efficiently.

\section{CONCLUSIONS}

A multi-level visual tracking algorithm is proposed based on MS algorithm, CS algorithm and EKF estimator. A MATLAB based simulation is developed for determining the proposed multi-level visual tracking algorithm performance. The simulation is tested and observed the proposed algorithm performance. The simulated results prove that the proposed multi-level visual tracking algorithm identifies and tracks the GMT efficiently. 
TABLE I. COMPUTED ERROR: MS ALGORITHM, CS ALGORITHM, EKF ESTIMATOR AND PROPOSED MULTI-LEVEL VISUAL TRACKING ALGORITHM (IN PIXELS).

\begin{tabular}{|c|c|c|c|c|c|c|c|c|c|c|c|c|c|c|c|c|c|c|}
\hline \multirow{3}{*}{\begin{tabular}{|c} 
Frame \\
No.
\end{tabular}} & \multicolumn{2}{|c|}{$\begin{array}{c}\text { GMT } \\
(x, y) \\
(1)\end{array}$} & \multicolumn{2}{|c|}{\begin{tabular}{|c} 
Mean-Shift \\
$(U, V)$ \\
$(2)$
\end{tabular}} & \multicolumn{2}{|c|}{$\begin{array}{c}\text { Cam-Shift } \\
(U, V) \\
(3)\end{array}$} & \multicolumn{2}{|c|}{$\begin{array}{c}\text { EKF } \\
(U, V) \\
(4)\end{array}$} & \multicolumn{2}{|c|}{$\begin{array}{c}\text { AVTS } \\
(U, V) \\
(5)\end{array}$} & \multicolumn{2}{|c|}{\begin{tabular}{|c} 
Mean-Shift \\
Error \\
$(1-2)$
\end{tabular}} & \multicolumn{2}{|c|}{\begin{tabular}{|c} 
Cam-Shift \\
Error \\
$(\mathbf{1 - 3 )}$
\end{tabular}} & \multicolumn{2}{|c|}{$\begin{array}{l}\text { EKF } \\
\text { Error } \\
(1-4)\end{array}$} & \multicolumn{2}{|c|}{$\begin{array}{l}\text { AVTS } \\
\text { Error } \\
\text { (1-5) }\end{array}$} \\
\hline & & Pos Y & & & & Pos V & & & & & & Pos & & & & Pos & Pos $\mathrm{X}$ & POSY \\
\hline & 382 & 232 & 357 & 205 & 361 & 208 & 364 & 212 & 367 & 215 & 25 & 27 & 21 & 24 & 18 & 20 & 15 & 17 \\
\hline 2 & 385 & 236 & 361 & 206 & 365 & 214 & 368 & 217 & \begin{tabular}{|l|l|}
3711 \\
\end{tabular} & 221 & 24 & 30 & 20 & 22 & 17 & 19 & 14 & 15 \\
\hline 3 & 388 & 233 & 366 & 208 & 368 & 211 & 372 & 215 & \begin{tabular}{|l|l|}
374 \\
\end{tabular} & 218 & 22 & 25 & 20 & 22 & 16 & 18 & 14 & 15 \\
\hline 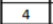 & 388 & 231 & \begin{tabular}{|l|l|}
370 \\
\end{tabular} & 210 & 370 & 211 & 372 & 214 & \begin{tabular}{|l|l|}
374 \\
\end{tabular} & 218 & 18 & 21 & 18 & 20 & 16 & 17 & 14 & 13 \\
\hline 5 & 395 & 237 & 72 & 211 & 378 & 2217 & 378 & 219 & \begin{tabular}{|l|l}
380 \\
\end{tabular} & 225 & 23 & 26 & 17 & 20 & 17 & 18 & 15 & 12 \\
\hline 6 & 392 & 238 & 375 & 214 & 376 & 219 & 379 & 224 & \begin{tabular}{|l|l}
3880 \\
\end{tabular} & 227 & 17 & 24 & 16 & \begin{tabular}{|l|}
19 \\
\end{tabular} & 13 & 14 & 12 & 11 \\
\hline 7 & 395 & 237 & 374 & 215 & 380 & 220 & 381 & 223 & \begin{tabular}{|l|l}
382 \\
\end{tabular} & 225 & 21 & 22 & 15 & 17 & 14 & 14 & 13 & 12 \\
\hline 8 & 392 & 238 & \begin{tabular}{|l|}
376 \\
\end{tabular} & 217 & 378 & \begin{tabular}{|l}
221 \\
\end{tabular} & 380 & 225 & \begin{tabular}{|l|l}
382 \\
\end{tabular} & 227 & 16 & \begin{tabular}{|l|}
21 \\
\end{tabular} & 14 & \begin{tabular}{|l|}
17 \\
\end{tabular} & 12 & 13 & 10 & 11 \\
\hline 9 & 390 & 238 & 78 & 218 & 377 & 2222 & 380 & 225 & \begin{tabular}{|l|l}
381 \\
\end{tabular} & 228 & & 20 & 13 & 16 & 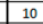 & 13 & & 10 \\
\hline 10 & \begin{tabular}{|c|}
391 \\
\end{tabular} & 239 & 381 & 221 & 379 & 224 & 382 & 227 & \begin{tabular}{|l|l}
383 \\
\end{tabular} & \begin{tabular}{|l|}
229 \\
\end{tabular} & 10 & \begin{tabular}{|l|}
18 \\
\end{tabular} & 12 & 15 & 9 & 12 & 8 & 10 \\
\hline 11 & \begin{tabular}{|l|l|}
389 \\
\end{tabular} & 240 & \begin{tabular}{|l|l|}
383 \\
\end{tabular} & 224 & 378 & 226 & 381 & 228 & \begin{tabular}{|l|l}
382 \\
\end{tabular} & 232 & 6 & \begin{tabular}{|l|}
16 \\
\end{tabular} & 11 & 14 & 8 & 12 & 7 & 8 \\
\hline 12 & \begin{tabular}{|l|l|}
387 \\
\end{tabular} & 240 & \begin{tabular}{|l|}
384 \\
\end{tabular} & 225 & 377 & 227 & 380 & 230 & \begin{tabular}{|l}
382 \\
\end{tabular} & 233 & 3 & 15 & 10 & 13 & 7 & 10 & 5 & 7 \\
\hline 13 & 386 & 241 & 385 & 227 & 376 & 228 & 380 & \begin{tabular}{|l}
231 \\
\end{tabular} & \begin{tabular}{|l|l|}
381 \\
\end{tabular} & 235 & & \begin{tabular}{|l|}
14 \\
\end{tabular} & & & & 10 & & 6 \\
\hline 14 & \begin{tabular}{|l|}
387 \\
\end{tabular} & 242 & 386 & 228 & 378 & 230 & 381 & 232 & \begin{tabular}{|l|l}
3882 \\
\end{tabular} & 236 & 1 & \begin{tabular}{|l|}
14 \\
\end{tabular} & 9 & \begin{tabular}{|l|}
12 \\
\end{tabular} & 6 & 10 & 5 & 6 \\
\hline 15 & 387 & 242 & \begin{tabular}{|l}
388 \\
\end{tabular} & 230 & 378 & \begin{tabular}{|l}
231 \\
\end{tabular} & 382 & 234 & \begin{tabular}{|l|l|}
383 \\
\end{tabular} & 238 & -1 & \begin{tabular}{|l|}
12 \\
\end{tabular} & 9 & \begin{tabular}{|l|}
11 \\
\end{tabular} & 5 & 8 & 4 & 4 \\
\hline 16 & 387 & 242 & \begin{tabular}{|l|}
390 \\
\end{tabular} & 234 & 379 & 232 & 382 & 235 & \begin{tabular}{|l|l}
383 \\
\end{tabular} & \begin{tabular}{|l|}
237 \\
\end{tabular} & -3 & 8 & 8 & \begin{tabular}{|l|}
10 \\
\end{tabular} & 5 & 7 & 4 & 5 \\
\hline 17 & \begin{tabular}{|l|}
386 \\
\end{tabular} & 243 & \begin{tabular}{|l|}
391 \\
\end{tabular} & 235 & 380 & 233 & 381 & \begin{tabular}{|l}
236 \\
\end{tabular} & \begin{tabular}{|l|l}
382 \\
\end{tabular} & 238 & -5 & 8 & 6 & 10 & 5 & 7 & 4 & 5 \\
\hline 18 & 386 & 243 & 390 & 237 & 380 & 234 & 381 & \begin{tabular}{|l|l|}
237 \\
\end{tabular} & \begin{tabular}{|l|l}
383 \\
\end{tabular} & \begin{tabular}{|l|}
239 \\
\end{tabular} & 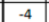 & & & & & & 3 & 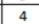 \\
\hline 19 & 384 & 244 & \begin{tabular}{|l|}
392 \\
\end{tabular} & 238 & 378 & 235 & 380 & \begin{tabular}{|l|l}
237 \\
\end{tabular} & \begin{tabular}{|l|l|}
381 \\
\end{tabular} & 240 & -8 & 6 & 6 & 9 & 4 & 7 & 3 & 4 \\
\hline 20 & 385 & 245 & 393 & 240 & 379 & 236 & 381 & 238 & 382 & 241 & -8 & 5 & 6 & 9 & 4 & 7 & 3 & 4 \\
\hline 21 & 385 & 248 & \begin{tabular}{|l|}
395 \\
\end{tabular} & 241 & 380 & 239 & 381 & \begin{tabular}{|l|l}
240 \\
\end{tabular} & \begin{tabular}{|l}
382 \\
\end{tabular} & 244 & -10 & 7 & 5 & 9 & 4 & 8 & 3 & 4 \\
\hline 22 & 384 & 247 & \begin{tabular}{|c|}
396 \\
\end{tabular} & 242 & 379 & 240 & 380 & \begin{tabular}{|l|l|}
241 \\
\end{tabular} & \begin{tabular}{|l|l|}
381 \\
\end{tabular} & 243 & 12 & 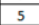 & & 7 & & 6 & 3 & 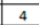 \\
\hline 23 & 385 & 249 & 398 & 243 & 380 & 241 & 381 & 243 & \begin{tabular}{|l|l|}
381 \\
\end{tabular} & 245 & \begin{tabular}{|l|}
-13 \\
\end{tabular} & 6 & 5 & 8 & 4 & & 4 & 4 \\
\hline 24 & 385 & 250 & \begin{tabular}{|l|}
399 \\
\end{tabular} & 247 & 380 & 242 & 381 & \begin{tabular}{|l|l|}
244 \\
\end{tabular} & \begin{tabular}{|l|l}
3882 \\
\end{tabular} & 246 & \begin{tabular}{|c|}
-14 \\
\end{tabular} & 3 & 5 & 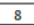 & 4 & 6 & 3 & 4 \\
\hline 25 & 386 & 250 & 400 & 250 & 381 & 243 & 382 & 245 & \begin{tabular}{|l|l}
383 \\
\end{tabular} & 246 & \begin{tabular}{l|}
-14 \\
\end{tabular} & 0 & 5 & 7 & 4 & 5 & 3 & 4 \\
\hline 26 & \begin{tabular}{|l|l|}
387 \\
\end{tabular} & 253 & 401 & 248 & 383 & 247 & 383 & \begin{tabular}{|l}
247 \\
\end{tabular} & \begin{tabular}{|l|l|}
384 \\
\end{tabular} & 250 & \begin{tabular}{l|l|}
-14 \\
\end{tabular} & 5 & 4 & 6 & 4 & 6 & 3 & 3 \\
\hline 27 & 388 & 255 & 402 & 250 & 384 & 249 & 384 & 249 & \begin{tabular}{|l|l}
385 \\
\end{tabular} & 252 & -14 & 5 & 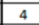 & 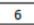 & & & & 3 \\
\hline 28 & \begin{tabular}{|l|l|}
300 \\
\end{tabular} & \begin{tabular}{|l|l|}
2556 \\
\end{tabular} & \begin{tabular}{|l}
402 \\
\end{tabular} & \begin{tabular}{|l|}
251 \\
\end{tabular} & 384 & \begin{tabular}{|l|}
251 \\
\end{tabular} &  & 252 & \begin{tabular}{|l}
380 \\
385
\end{tabular} & 253 & -16 & 5 & 3 & 5 & 2 & 4 & 2 & 3 \\
\hline 29 & 388 & \begin{tabular}{|l|l|}
257 \\
\end{tabular} & 404 & 251 & 385 & 253 & 386 & 254 & \begin{tabular}{|l|l|}
386 \\
\end{tabular} & 254 & -16 & 6 & 3 & 4 & 2 & 3 & 2 & 3 \\
\hline 30 & \begin{tabular}{|l|l|}
389 \\
\end{tabular} & 258 & \begin{tabular}{|l|l}
404 \\
\end{tabular} & 254 & 387 & 254 & 387 & 255 & \begin{tabular}{|l|l}
387 \\
\end{tabular} & 256 & -15 & 4 & 2 & 4 & 2 & 3 & 2 & 2 \\
\hline 31 & 391 & 261 & 405 & 255 & 389 & 258 & 389 & 258 & 390 & 259 & 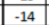 & 6 & & 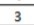 & 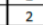 & & 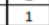 & 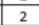 \\
\hline 32 & 393 & 262 & 407 & 256 & 391 & 260 & 391 & & 392 & 261 & -14 & & & & & & & 1 \\
\hline
\end{tabular}

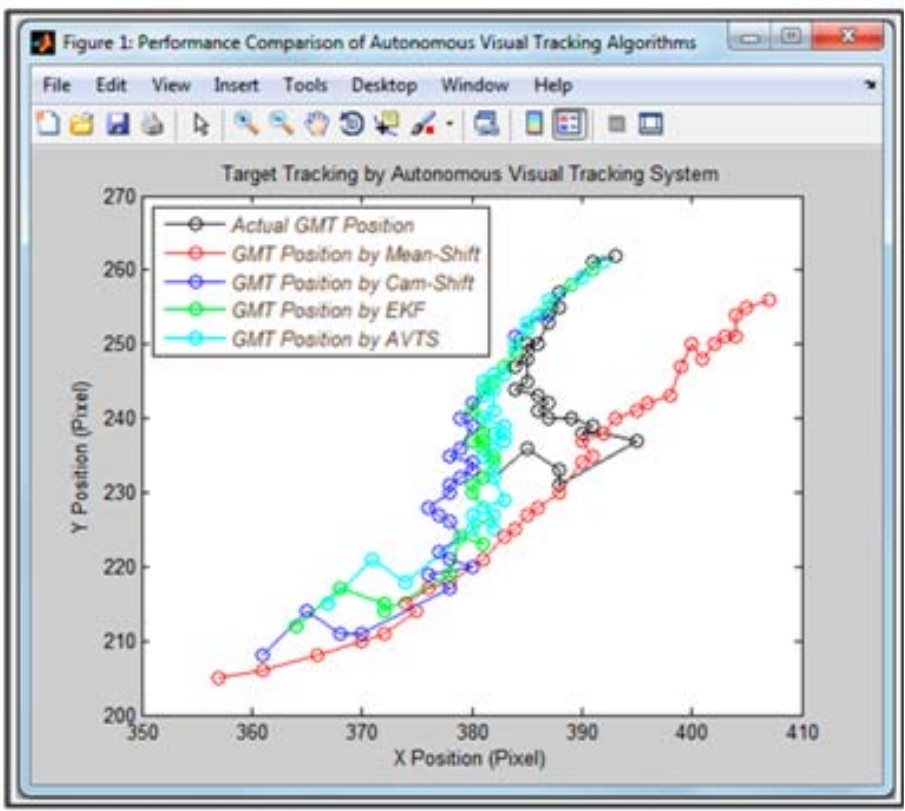

Fig. 7. Performance comparison: MS algorithm, CS algorithm, EKF estimator and Proposed Multi-level Visual Tracking Algorithm (in pixels).

TABLE II. MS ALGORITHM, CS ALGORITHM, EKF ESTIMATOR AND PROPOSED MULTI-LEVEL VISUAL TRACKING ALGORITHM EXECUTION TIME (IN MS).

\begin{tabular}{|c||l||c||}
\hline No. & \multicolumn{1}{|c|}{ Algorithm/Estimator } & $\begin{array}{c}\text { Time } \\
\text { (in msec) }\end{array}$ \\
\hline \hline 1. & MS Algorithm & 17.408675 \\
\hline \hline 2. & CS Algorithm & 19.523104 \\
\hline \hline 3. & EKF Estimator & 24.104903 \\
\hline \hline 4. & $\begin{array}{l}\text { Multi-level Visual Tracking } \\
\text { Algorithm }\end{array}$ & 15.938718 \\
\hline
\end{tabular}

\section{REFERENCES}

[1] K. Narsimlu, Dr. T. V. Rajini Kanth, and Dr. Devendra Rao Guntupalli, "A Comparative Study on Image Fusion Algorithms for Avionics Applications,” International Journal of Advanced Engineering and Global Technology (IJAEGT), ISSN No: 2309-4893, vol. 2, no. 4, April 2014, pp. 616-621.

[2] K. Narsimlu, Dr. T. V. Rajini Kanth, and Dr. Devendra Rao Guntupalli, "An Experimental Study of the Autonomous Visual Target Tracking Algorithms for Small Unmanned Aerial Vehicles,” 1st International Conference on Rough Sets and Knowledge Technologies-2014 (ICRSKT-2014), 09-11 Nov. 2014, Elsevier Publications, pp. 52-59.

[3] K. Narsimlu, Dr. T. V. Rajini Kanth, and Dr. Devendra Rao Guntupalli, "Autonomous Visual Tracking with Extended Kalman Filter Estimator for Micro Aerial Vehicles,” Fifth International Conference on Fuzzy and Neuro Computing2015 (FANCCO-2015), 16-19 Dec. 2015, Springer Publications, pp. 31-42.

[4] K. Narsimlu, Dr. T. V. Rajini Kanth, and Dr. Devendra Rao Guntupalli, "A Mean-Shift Algorithm Based Autonomous Visual Tracking for Micro Aerial Vehicles,” International Journal of Recent Trends in Engineering \& Research (IJRTER), vol. 2, no. 4, pp. 362-369, Apr. 2016.

[5] K. Narsimlu, Dr. T. V. Rajini Kanth, Dr. Devendra Rao Guntupalli, and Anil Kuvvarapu "An Autonomous Visual Tracking Algorithm Based on Mean-Shift Algorithm and Extended Kalman Filter Estimator,” International Journal of Innovative Computer Science \& Engineering (IJICSE), vol. 3, no. 2, pp. 14-23, Mar-Apr. 2016.

[6] K. Narsimlu, Dr. T. V. Rajini Kanth, Dr. Devendra Rao Guntupalli, and Anil Kuvvarapu "An Efficient Approach of Autonomous Visual Tracking for Micro Aerial Vehicles," International Journal of Recent Scientific Research (IJRSR), vol. 7, no. 6, pp. 11959-11964, Jun. 2016.

[7] K. Narsimlu, Dr. T. V. Rajini Kanth, Dr. Devendra Rao Guntupalli, and Anil Kuvvarapu "A Hybrid Autonomous Visual Tracking Algorithm for Micro Aerial Vehicles," International Journal of Engineering Sciences \& Research Technology (IJESRT), vol. 5, no. 8, pp. 524-535, Aug. 2016.

[8] I. Cohen, and G. Medioni, "Detecting and tracking moving objects in video from and airborne observer," in IEEE Image Understanding Workshop, pp. 217-222, 1998.

[9] Yau, W. G. Fu, L-C., and Liu, D, "Design and Implementation of Visual Servoing System for Realistic Air Target Tracking," in Proc. of the IEEE International Conference on Robotics and Automation - ICRA, vol. 1, 2001, pp. 229-234.

[10] Johnson, E. N. Schrage, and D. P, "The Georgia Tech Unmanned Aerial Research Vehicle: GTMax," Aug 11-14, Austin, Texas, 2003.

[11] Vladimir N. Dobrokhodov, Isaac I. Kaminer, Kevin D. Jones, and Reza Ghabcheloo, "Vision-Based Tracking and Motion Estimation for Moving targets using Small UAVs," in the American Control Conference, Minneapolis, USA. June 1416, 2006.

[12] P. Theodorakopoulos, and S. Lacroix, "A strategy for tracking a ground target with a uav," in Intelligent Robots and Systems, 2008. IROS 2008. IEEE/RSJ International Conference, pp. 1254-1259, 2008.

[13] Mingfeng Zhang, and Hugh H.T. Liu, "Vision-Based Tracking and Estimation of Ground Moving Target Using Unmanned Aerial Vehicle," in the American Control Conference, Marriott Waterfront, Baltimore, MD, USA. June 30-July 02, 2010.

[14] Zhiyuan Li, Naira Hovakimyan, Vladimir Dobrokhodov, and Isaac Kaminer, "Vision-based Target Tracking and Motion Estimation Using a Small UAV," in the 49th IEEE Conference on Decision and Control, Atlanta, GA. December 15-17, 2010. 
[15] Amr Awwad El-Kalubi, Rui Zhou, and Haibo Sun, "VisionBased real time guidance of UAV," in International Conference on Management and Service Science - MASS, Wuhan, 12-14 August, 2011.

[16] Zhekui Xin, Yongchun Fang, and Bin Xian, "An on-board Pan-tilt Controller for Ground Target Tracking Systems," in IEEE International Conference on Control Applications (CCA), CO, USA. September 28-30, 2011.

[17] Raja, Akshay Srivastava, Abhishek Dwivedi, and Himanshu Tiwari. (2014). Vision Based Tracking for Unmanned Aerial Vehicle. Advances in Aerospace Science and Applications. ISSN 2277-3223, vol. 4, no. 1, Research India Publications, pp. 59-64, 2014.

[18] Xun Wang, Huayong Zhu, Daibing Zhang, Dianle Zhou, and Xiangke Wang. (2014, Aug.). Vision-based Detection and Tracking of a Mobile Ground Target Using a Fixed-wing UAV. International Journal of Advanced Robotic Systems.

[19] Hu, W., Tan, T., Wang, L., and Maybank, S. (2004). A Survey on Visual Surveillance of Object Motion and Behaviors. IEEE Trans. on Syst., Man, Cybern. C, Appl. Rev. 34, no. 3, pp. 334-352.

[20] Yilmaz, A., Javed, O., and Shah, M. (2006, Dec.). Object tracking: A survey. ACM Computing Surveys, vol. 38, no. 4, Article 13

[21] Kinjal A Joshi, and Darshak G. Thakore. (2012, Jul.). A Survey on Moving Object Detection and Tracking in Video Surveillance System. International Journal of Soft Computing and Engineering (IJSCE), vol. 2, no. 3.

[22] Chandrashekhar D. Badgujar, and Dipali P.Sapkal. (2012, Oct.). A Survey on Object Detect, Track and Identify Using Video Surveillance. IOSR Journal of Engineering (IOSRJEN), vol. 2, no. 10, pp. 71-76.

[23] Barga Deori, and Dalton Meitei Thounaojam. (2014, Jul.). A Survey On Moving Object Tracking In Video. International Journal on Information Theory (IJIT), vol. 3, no. 3.

[24] Comaniciu D, and Meer P, "Mean Shift Analysis and Applications," in Proc. of the IEEE International Conference on Computer Vision (ICCV), 1999, pp. 1197-1203.

[25] Dorin Comaniciu, Visvanathan Ramesh, and Peter Meer, "Real-Time Tracking of Non-Rigid Objects using Mean Shift," in Proc. of the Conference on Computer Vision and Pattern Recognition, vol. 2, 2000, pp. 142-149.

[26] Dorin Comaniciu, and Peter Meer. (2002, May). Mean Shift: A Robust Approach Toward Feature Space Analysis. IEEE Trans. PAMI, vol. 24, no. 5.

[27] R. T. Collins, "Mean-shift Blob Tracking through Scale Space," in Proc. of IEEE Conference on Computer Vision and Pattern Recognition, 2003, pp. 234-240.

[28] Leung, A., and Gong, S, "Mean-Shift Tracking with Random Sampling,” BMVC, 2, 2006, pp. 729-738.

[29] Sylvain Paris, and Frédo Durand, "A Topological Approach to Hierarchical Segmentation using Mean Shift," in IEEE Computer Society Conference on Computer Vision and Pattern Recognition (CVPR 2007), 2007, pp.18-23.

[30] Rahul Mishra, Mahesh K. Chouhan, and Dhiiraj Nitnawwre, "Object Tracking By Adaptive Mean Shift With Kernel Based Centroid Method,” in IJCSC vol. 3, no. 1, January-June 2012, pp. 39-42.

[31] Dorin Comaniciu, Visvanathan Ramesh, and Peter Meer. (2003, May). Kernel-Based Object Tracking. IEEE Transactions on Pattern Analysis and Machine Intelligence, vol. 25, no. 5, pp. 564-577.

[32] Allen, J.G., Xu, R.Y.D., Jin, J.S, "Object Tracking Using CamShift Algorithm and Multiple Quantized Feature Spaces,” Proc. of the Pan-Sydney area Workshop on Visual Information Processing, pp. 3-7, 2004.

[33] Stolkin, R., Florescu, I., Kamberov, G, “An Adaptive Background Model for CamShift Tracking with a Moving Camera," Proc. of the 6th International Conference on Advances in Pattern Recognition, 2007.
[34] Intel Corporation: OpenCV Reference Manual v2.1. March 18, 2010.

[35] Emami, E, Fathy, M, "Object Tracking Using Improved CAMShift Algorithm Combined with Motion Segmentation," 2011.

[36] Li Zhu, Tao Hu, "Research of CamShift Algorithm to Track Motion Objects," in TELKOMNIKA, vol. 11, no. 8, August 2013, pp. 4372-4378.

[37] Kalman, R.E. (1960), “A New Approach to Linear Filtering and Prediction Problems," Transactions of the ASME Journal of Basic Engineering, vol. 82, pp. 35-45.

[38] Kalman, R. E., Bucy R. S. (1961), "New Results in Linear Filtering and Prediction Theory," Transactions of the ASME Journal of Basic Engineering, vol. 83, pp. 95-107.

[39] G. Welch and G. Bishop (2001), "An Introduction to the Kalman Filter,” Proceedings of SIGGRAPH, pp. 19-24.

[40] F. Janabi, and M. Marey (2010), "A Kalman filter based method for pose estimation in visual servoing," IEEE Trans. Robotics, vol. 26, no. 5, pp. 939-947.

[41] B. Torkaman and M. Farrokhi (2012), “A Kalman-FilterBased Method for Real-Time Visual Tracking of a Moving Object Using Pan and Tilt Platform,” International Journal of Scientific \& Engineering Research, vol. 3, no. 8.

[42] Sara Qazvini Abhari, Qazvin-Iran, and Towhid Zargar Ershadi, "Target Tracking Based on Mean Shift and KALMAN Filter with Kernel Histogram Filtering,” Computer and Information Science vol. 4, no. 2, March 2011, pp. 152160.

[43] Afef Salhi, Ameni Yengui Jammoussi (2012), “Object tracking system using Camshift, Meanshift and Kalman filter," World Academy of Science, Engineering and Technology.

[44] Ravi Kumar Jatoth, Sampad Shubhra, and Ejaz Ali, "Performance Comparison of Kalman Filter and Mean Shift Algorithm for Object Tracking,” I.J. Information Engineering and Electronic Business, 2013, 5, 17-24.

[45] Ashvini Kulkarni, and Manasi Vargantwar, "Video Based Tracking with Mean-Shift and Kalman Filter,” in International Journal of Engineering Research \& Technology (IJERT), vol. 3, no. 5, May, 2014, pp. 1271-1274.

[46] Zouguo Yan, Weiguo Liang, Haidong lv, "A Target Tracking Algorithm Based on Improved Camshift and UKF,” in Journal of Software Engineering and Applications, pp. 1065-1073, December, 2014.

[47] Shao-Fan Lien, Kuo-Hsien Hsia, and Juhng-Perng Su, "Moving Target Tracking based on CamShift Approach and Kalman Filter," Applied Mathematics \& Information Sciences, vol. 9, no. 1, Jan 2015, pp. 395-401.

[48] Gaurav.R.Desai, and Prof.K.R.Desai, "Hybrid Method For Moving Object Tracking Within A Video Sequence \& Occlusion Handling," International Journal of Advanced Technology in Engineering and Science, vol. 03, no. 03, March 2015, pp. 27-32.

[49] Derek Kingston, Randal Beard, Timothy McLain, Michael Larsen, Wei Ren, "Autonomous Vehicle Technologies For Small Fixed Wing Uavs," AIAA Journal of Aerospace, vol. 2, no. 1, pp. 92-108, 2003.

[50] Qadir, A., Neubert, J., and Semke, W. (2011, Mar.). On-Board Visual Tracking with Unmanned Aircraft System. AIAA Infotech@Aerospace conference, St. Louis, MO, pp. 28-31.

[51] Roozbeh Falah Ramezani, "Non-linear Modeling and Control of Unmanned Air Vehicle," M.Sc. thesis, Mechatronics Engineering, Sharjah, United Arab Emirates, May, 2012.

[52] Adam Ufford, "Development and Implementation of Guidance, Navigation and Control Systems for an Autonomous Air Vehicle," M.Sc. thesis, Department of Mechanical Engineering, Texas Tech University, August, 2009

[53] Amer A. KH. Al-Radaideh, "Guidance, Control and Trajectory Tracking of Small Fixed Wing Unmanned Aerial 
Vehicles (UAV's)," M.Sc. thesis, American University of Sharjah, April, 2009.

[54] Rivero Garcia, and Alfonso, “Gimbal Control,” M.Sc. thesis, School of Engineering, Cranfield University, August, 2012.

[55] Nicholas J. Brake, "Control System Development For Small Uav Gimbal," M.Sc. thesis, Department of Aerospace Engineering, California Polytechnic State University, San Luis Obispo, August, 2012.

[56] Diogo Filipe Cabral de Sousa Leite, "Target Tracking with Pan-Tilt-Zoom Cameras," M.Sc. thesis, Instituto Superior Technico, November, 2011.

[57] Haiyang Chao, Yongcan Cao, YangQuan Chen, "Autopilots for Small Fixed-Wing Unmanned Air Vehicles: A Survey," in
Proc. of the IEEE International Conference on Mechatronics and Automation, August 5 - 8, 2007, Harbin, China.

[58] Reed Siefert Christiansen, "Design of an Autopilot for Small Unmanned Aerial Vehicles," M.Sc. thesis, Department of Electrical and Computer Engineering, Brigham Young University, August, 2004.

[59] Ingrid Hagen Johansen, "Autopilot Design for Unmanned Aerial Vehicles," M.Sc. thesis, Department of Engineering Cybernetics, Norwegian University of Science and Technology, June 2012.

[60] UAV Vision Pty Ltd, [Online]. http://www.uavvision.com. 